\title{
Ultrasound-Guided Lateral Femoral Cutaneous Nerve Conduction Study
}

\author{
Bum Jun Park, MD, Eui Soo Joeng, MD, Jun Kyu Choi, MD, Seok Kang, MD, \\ Joon Shik Yoon, MD, PhD, Seung Nam Yang, MD, PhD
}

Department of Physical Medicine and Rehabilitation, Korea University Guro Hospital, Seoul, Korea

\begin{abstract}
Objective To verify the utility of the lateral femoral cutaneous nerve (LFCN) ultrasound-guided conduction technique compared to that of the conventional nerve conduction technique.

Methods Fifty-eight legs of 29 healthy participants (18 males and 11 females; mean age, $42.7 \pm 14.9$ years) were recruited. The conventional technique was performed bilaterally. The LFCN was localized by ultrasound. Crosssectional area (CSA) of the LFCN and the distance between the anterior superior iliac spine (ASIS) and the LFCN was measured. The nerve conduction study was repeated with the corrected cathode location. Sensory nerve action potential (SNAP) amplitudes of the LFCN were recorded and compared between the ultrasound-guided and conventional techniques.

Results Mean body mass index of the participants was $23.7 \pm 3.5 \mathrm{~kg} / \mathrm{m}^{2}$, CSA was $4.2 \pm 1.9 \mathrm{~mm}^{2}$, and the distance between the ASIS and LFCN was $5.6 \pm 1.7 \mathrm{~mm}$. The mean amplitude values were $6.07 \pm 0.52 \mu \mathrm{V}$ and $6.66 \pm 0.54 \mu \mathrm{V}$ using the conventional and ultrasound-guided techniques, respectively. The SNAP amplitude of the LFCN using the ultrasound-guided technique was significantly larger than that recorded using the conventional technique.

Conclusion Correcting the stimulation position using the ultrasound-guided technique helped obtain increased SNAP amplitude.
\end{abstract}

Keywords Ultrasound-guided, Nerve conduction study, Lateral femoral cutaneous nerve, Ultrasonography, Lumbosacral plexus

\section{INTRODUCTION}

The lateral femoral cutaneous nerve (LFCN) is a pure sensory nerve originating from the $\mathrm{L} 2$ and L3 roots. The

Received April 4, 2014; Accepted August 29, 2014

Corresponding author: Seung Nam Yang

Department of Physical Medicine and Rehabilitation, Korea University Guro Hospital, 148 Gurodong-ro, Guro-gu, Seoul 152-703, Korea

Tel: +82-2-2626-1500, Fax: +82-2-2626-1513, E-mail: snamyang@korea.ac.kr

(c) This is an open-access article distributed under the terms of the Creative Commons Attribution Non-Commercial License (http://creativecommons. org/licenses/by-nc/3.0) which permits unrestricted noncommercial use, distribution, and reproduction in any medium, provided the original work is properly cited.

Copyright $\odot 2015$ by Korean Academy of Rehabilitation Medicine
LFCN supplies sensation to the anterolateral aspect of the thigh [1] and courses lateral to the psoas muscle crossing the iliacus muscle. Around the anterior superior iliac spine (ASIS), the LFCN passes under the inguinal ligament and over the sartorius muscle into the thigh. The most common course of the LFCN is medial and inferior to the ASIS [2]. The nerve passes through the fascia and becomes a smaller branch about $10 \mathrm{~cm}$ inferior to the inguinal ligament [3].

A LFCN nerve conduction study (NCS) can be used to differentially diagnose meralgia paresthetica, upper lumbar radiculopathy, and lumbar plexopathy. However, an LFCN NCS can be hindered by the anatomic variability 
of its course around the ASIS and terminal branch, which results in inaccurate NCS results [3]. About one-quarter of the population has an anatomical variation in the LFCN [4]. About $19 \%$ of LFCNs passed lateral to the ASIS in a cadaveric study [5], and Aszmann et al. [6] reported that $4 \%$ of human anatomic specimens have a LFCN that passes lateral to the ASIS. Therefore, identifying the proximal location of the LFCN is important to obtain reliable LFCN NCS results.

Ultrasound is useful for assessing peripheral nerves and noninvasively observing small nerve anatomy and pathology. Refinements in ultrasound have enabled the detection of small peripheral nerves, which leads to a better understanding of the locations of small nerves and makes ultrasound useful to localize the LFCN [7-12].

Our hypothesis was that correcting the stimulation site using ultrasound during a LFCN NCS would increase sensory nerve action potential (SNAP) amplitude and elicit a better response compared with that of a conventional LFCN NCS. The primary objective was to compare the SNAP value of an ultrasound-guided LFCN NCS with SNAP of a conventional LFCN NCS. The secondary objective was to establish normal LFCN reference values for ultrasound.

\section{MATERIALS AND METHODS}

\section{Subjects}

Twenty-nine healthy volunteers (18 men and 11 women; age range, 23-68 years; mean age, 42.7 years) participated in this study. All participants provided informed consent. This protocol was approved by the regional Institutional Review Board of Korea University Guro Hospital. Patients with a history of pelvic surgery, thigh area trauma, hereditary neuropathy, diabetes, amyloidosis, chronic renal failure, or chronic alcoholism were excluded. General characteristics of age, sex, height, weight, and leg length were evaluated.

\section{Conventional NCS}

The conventional LFCN NCS was performed bilaterally. The E1 electrode was placed $12 \mathrm{~cm}$ distal to the ASIS along an imaginary line between the ASIS and the lateral border of the patella, and the E2 electrode was placed 4 $\mathrm{cm}$ distal to the E1. Stimulation was performed with a stimulator placed $1 \mathrm{~cm}$ medial to the ASIS. Stimulus mag- nitude was supramaximal intensity. Current intensity was increased until the amplitude of the recorded potential reached a plateau at supramaximal stimulation. The anode was rotated when necessary, and five responses were averaged after supramaximal stimulation was achieved. Negative peak latency and baseline to peak amplitude of the SNAP were recorded. 'No response' was recorded if there was no evoked response following maximum stimulation intensity.

Skin temperature was monitored and maintained $>32^{\circ} \mathrm{C}$ at the thigh. The NCS was carried out using the Viking Select System (Nicolet Biomedical, Madison, WI, USA) and the following equipment settings: sensitivity, $10 \mu \mathrm{V} /$ division; sweep speed, $1 \mathrm{~ms} /$ division; and bandwidth, 20-3,000 Hz.

\section{Ultrasound examination}

After the conventional NCS, the LFCN was examined with ultrasound around the ASIS performed by a skilled physiatrist with neuromuscular ultrasound experience. The ultrasound probe was placed in a transverse plane 2-3 cm distal to the ASIS (Fig. 1). We found the LFCN in the intermuscular space between the tensor fascia lata and the sartorius muscle distal to the ASIS, after which the nerve was traced more cranially. The LFCN was locat-

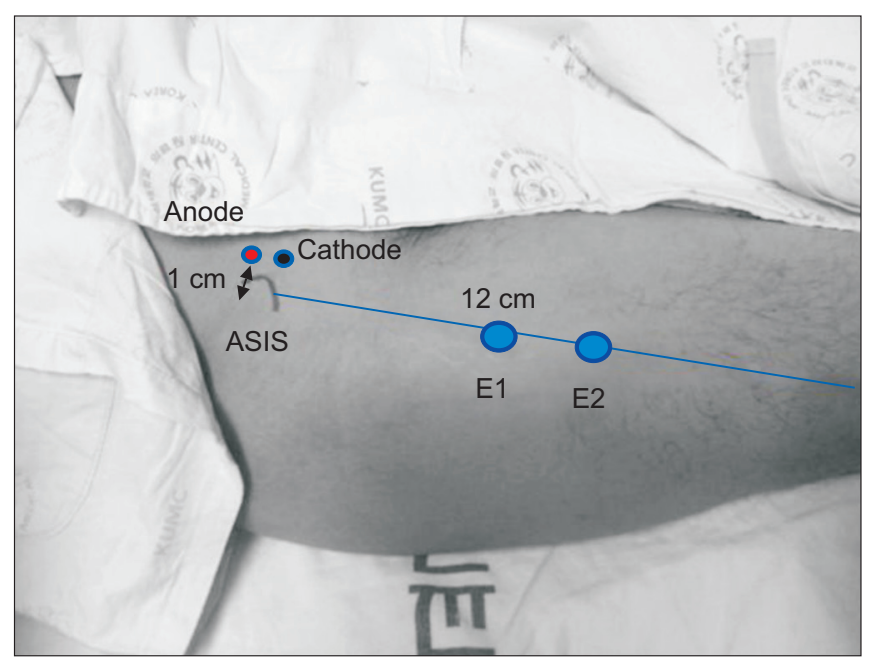

Fig. 1. The E1 electrode was placed $12 \mathrm{~cm}$ distal to the anterior superior iliac spine (ASIS) along an imaginary line between the ASIS and the lateral border of the patella. The E2 electrode was placed $4 \mathrm{~cm}$ distal to the E1. Stimulation was performed with a stimulator placed $1 \mathrm{~cm}$ medial to the ASIS. 
ed in the space between the two fascia layers (fascia lata and the fascia iliaca) at the level of the ASIS. Then, we analyzed the morphology of the nerve, cross-sectional area (CSA), and distance from the ASIS. Nerve CSA was measured by directly tracing just inside the hyperechoic rim of the nerve and was measured three times to increase accuracy. The color Doppler ultrasound mode was used before tracing the nerve to prevent overestimating the nerve CSA by including blood vessels. An effort was made to minimize additional probe pressure on the nerve. The LFCN location was marked on the skin using a clip pin as the ultrasound-guided NCS stimulation site (Fig. 2). Ultrasound was performed using the HD15 Ultrasound System (Philips, Bothell, WA, USA) and a 7-12 MHz linear array transducer. The ultrasound settings were optimized for nerve imaging, such as frequency, depth, and focal zone.

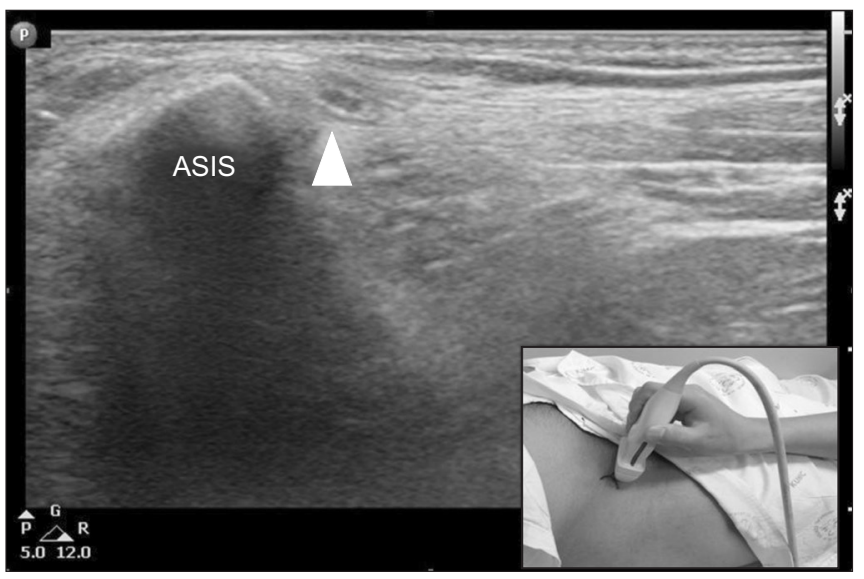

Fig. 2. Sonographic findings of the lateral femoral cutaneous nerve at the anterior superior iliac spine (ASIS) (arrowhead).

\section{Ultrasound-guided NCS}

The ultrasound-guided NCS was performed using the corrected stimulation position determined by an ultrasound examination (Fig. 3). The E1 and E2 recording electrodes were placed at the same locations as those used for a conventional NCS ( $12 \mathrm{~cm}$ distal to the ASIS).

\section{Statistical analysis}

Data were analyzed using SAS ver. 9.2 (SAS Institute, Cary, NC, USA) and SPSS ver. 20.0 (IBM SPSS Inc., Armonk, NY, USA). The general linear model with unstructured covariance was used to analyze the results. SNAP latency and LFCN amplitude were compared between the conventional and ultrasound-guided techniques. Correlations between demographic data and SNAP latency and amplitude were also evaluated using the general linear model.

\section{RESULTS}

All 29 subjects completed the study successfully (Table 1). Fifty-seven LFCNs were identified by a sensory NCS. No response was elicited in one leg. The general characteristics of the subjects are presented in Table 1.

Table 1. Subject characteristics

\begin{tabular}{lc}
\hline \multicolumn{1}{c}{ Parameter } & Value \\
\hline Sex $($ male:female $)$ & $18: 11$ \\
Age $(\mathrm{yr})$ & $42.7 \pm 14.9$ \\
Height $(\mathrm{cm})$ & $169.1 \pm 10.7$ \\
Leg length $(\mathrm{cm})$ & $83.8 \pm 5.2$ \\
\hline Body mass index $\left(\mathrm{kg} / \mathrm{m}^{2}\right)$ & $23.7 \pm 3.5$ \\
\hline
\end{tabular}

Values are presented as mean \pm standard deviation.
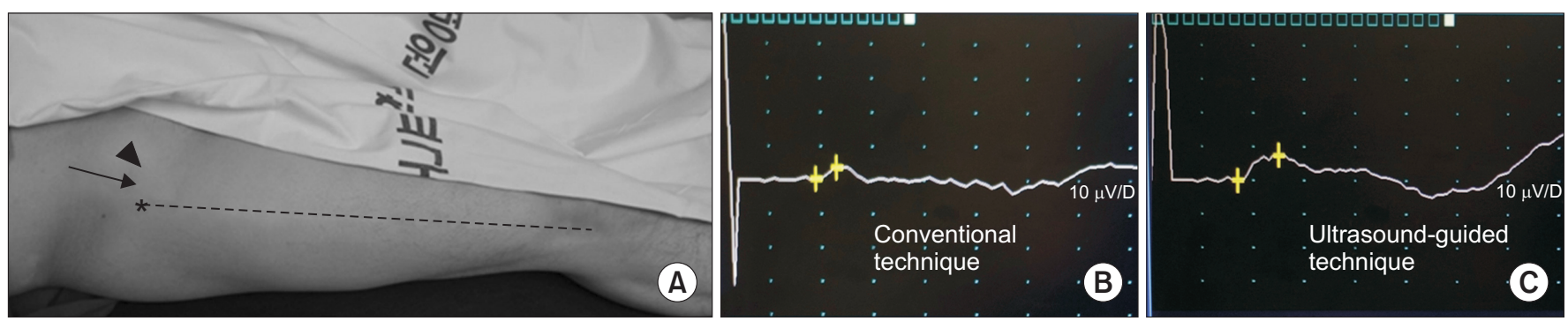

Fig. 3. (A) Photograph of the stimulation site for the lateral femoral cutaneous nerve conduction study using the conventional technique (arrow) and the ultrasound-guided technique (arrowhead) around the anterior superior iliac spine (ASIS) (asterisks). (B) Sensory nerve action potential (SNAP) of the lateral femoral cutaneous nerve (LFCN) using the conventional technique. (C) SNAP of the LFCN using the ultrasound-guided technique. 
Table 2. Least square means for the lateral femoral cutaneous nerve conduction study

\begin{tabular}{lccc}
\hline & Conventional & $\begin{array}{c}\text { Ultrasound- } \\
\text { guided }\end{array}$ & p-value \\
\hline Latency $(\mathrm{ms})$ & $2.67 \pm 0.08$ & $2.65 \pm 0.08$ & 0.5597 \\
Amplitude $(\mu \mathrm{V})$ & $6.07 \pm 0.52$ & $6.66 \pm 0.54$ & $0.0296^{*}$ \\
\hline
\end{tabular}

Values are presented as mean \pm standard error. ${ }^{*} \mathrm{p}<0.05$.

\section{Conventional vs. ultrasound-guided NCS}

The SNAP response was obtained bilaterally in 28 of 29 subjects during the conventional NCS. The estimated mean negative peak latency and SNAP amplitude values were $2.67 \pm 0.08 \mathrm{~ms}$ and $6.07 \pm 0.52 \mu \mathrm{V}$, respectively. An appropriate biphasic response was elicited in 28 of 29 subjects during the ultrasound-guided NCS. The estimated mean negative peak latency and SNAP amplitude values were $2.65 \pm 0.08 \mathrm{~ms}$ and $6.66 \pm 0.54 \mu \mathrm{V}$. Significant differences were observed between the SNAP amplitudes measured during the ultrasound-guided NCS and the conventional NCS. However, no significant difference in SNAP latency was observed between the conventional and ultrasound-guided NCS (Table 2).

\section{Sonographic data}

The CSA of the LFCN was $4.2 \pm 1.9 \mathrm{~mm}^{2}$, and the mean distance between the ASIS and the LFCN was 5.6 \pm 1.7 $\mathrm{mm}$. No significant correlation was observed between the ultrasound findings and age, sex, height, weight, body mass index (BMI), or leg length.

\section{DISCUSSION}

SNAP amplitude differed significantly between the ultrasound-guided NCS and the conventional NCS in our study. This result demonstrates that correcting the stimulation site by ultrasound improved reliability of the LFCN NCS. Previous studies have attempted to evaluate the reliability of a LFCN ultrasound-guided NCS. Boon et al. [13] performed an ultrasound-guided LFCN NCS focusing on the exact location of the recording electrode in obese subjects (BMI, $27.2 \pm 4.8 \mathrm{~kg} / \mathrm{m}^{2}$ ). They obtained the LFCN SNAP in 46 of 50 subjects using a conventional NCS and in 49 of 50 subjects using an ultrasound-guided NCS. LFCN SNAP was better using the ultrasound-guided NCS, with a mean value of $9.3 \pm 5.5 \mu \mathrm{V}$, compared with 7.0 \pm 3.8 $\mu \mathrm{V}$ by the conventional NCS. They thought that correcting the stimulation site had less of an effect. Although little difference was observed in the mean SNAP amplitude value, our ultrasound-guided NCS results were significantly different compared with those of the conventional NCS. Furthermore, a previous study evaluated the reliability of an LFCN nerve conduction technique in obese patients (mean BMI, $27.2 \mathrm{~kg} / \mathrm{m}^{2}$ ), whereas our 29 normal subjects had a lower BMI $\left(23.7 \mathrm{~kg} / \mathrm{m}^{2}\right)$. Fewer Asians, including Koreans, have a high BMI, so our results may be more significant for the Asian population. These results suggest that not only the obese and symptom-related population but also those with a lower BMI and asymptomatic subjects require a corrected stimulation position using ultrasound-guided NCS to obtain better SNAP amplitude. The reason for the small difference in mean SNAP amplitude was the low BMI of our subjects. LFCN depth is shallower in subjects with a lower BMI, and it is relatively easy to find an anatomic landmark, such as the ASIS, and it is easy to predict the LFCN path, so the conventional NCS obtained relatively good results.

Another reason for correcting the stimulation site is anatomical variability at the level of the ASIS. Aszmann et al. [6] reported five different LFCN courses in the pelvic area. LFCN location is reportedly relatively far from the ASIS in types A (4\%) and E (20\%), but just medial to the ASIS in types B (27\%), C (23\%), and D (26\%). In our study, the distance between the ASIS and LFCN was $5 \mathrm{~mm}$, which was shorter than that in other studies. It seems that subjects with types B, C, and D were recruited by chance. Because our study was performed on healthy volunteers, type A subjects, who could be vulnerable to nerve compression, were not recruited.

The higher sensitivity observed when the results of multiple techniques are combined helps to detect patients with meralgia paresthetica. While time-consuming, combining both techniques helps overcome the possible limitations of either technique. In one study, the combination of somatosensory-evoked potential and sensory NCS provided more sensitive results [14]. We did not assess sensitivity or specificity of the ultrasoundguided NCS. However, we expect that the combination of somatosensory-evoked potential and ultrasound NCS is appropriate for detecting meralgia paresthetica.

Our study had several limitations. First, we did not localize the LFCN at the recording site. Because the best 
view of the LFCN was about $5 \mathrm{~cm}$ inferior to the ASIS, accurate sonographic observations were difficult at 12 $\mathrm{cm}$ distal. Furthermore, it would be time consuming if we used ultrasound to detect the LFCN at the recording site. Second, our study had a relatively small number of healthy subjects. Third, most of our subjects had a low BMI, so the role of ultrasound detection and correcting the stimulation site was limited, as the response may have been elicited by increasing intensity to induce volume conduction, which changed the stimulation site slightly. Fourth, the anatomical variation in which the LFCN crosses outside the iliac crest was not included in our study. Further studies are required to demonstrate the usefulness of our technique in subjects with an anatomical variation. Finally, we did not examine patients with meralgia paresthetica.

In conclusion, the reliability of a LFCN NCS was suspect due to anatomic variability around the ASIS. The ultrasound-guided LFCN NCS showed a significant difference in SNAP amplitude compared to that of the conventional NCS technique. The ultrasound-guided technique obtained higher SNAP amplitudes after correcting the stimulation position than that of the conventional technique.

\section{CONFLICT OF INTEREST}

No potential conflict of interest relevant to this article was reported.

\section{REFERENCES}

1. Patijn J, Mekhail N, Hayek S, Lataster A, van Kleef M, Van Zundert J. Meralgia paresthetica. Pain Pract 2011; 11:302-8.

2. Keegan JJ, Holyoke EA. Meralgia paresthetica. An anatomical and surgical study. J Neurosurg 1962;19:341-5.

3. Shin YB, Park JH, Kwon DR, Park BK. Variability in conduction of the lateral femoral cutaneous nerve. Muscle Nerve 2006;33:645-9.

4. de Ridder VA, de Lange S, Popta JV. Anatomical varia- tions of the lateral femoral cutaneous nerve and the consequences for surgery. J Orthop Trauma 1999; 13:207-11.

5. Kosiyatrakul A, Nuansalee N, Luenam S, Koonchornboon T, Prachaporn S. The anatomical variation of the lateral femoral cutaneous nerve in relation to the anterior superior iliac spine and the iliac crest. Musculoskelet Surg 2010;94:17-20.

6. Aszmann OC, Dellon ES, Dellon AL. Anatomical course of the lateral femoral cutaneous nerve and its susceptibility to compression and injury. Plast Reconstr Surg 1997;100:600-4.

7. Won SJ, Kim BJ, Park KS, Yoon JS, Choi H. Reference values for nerve ultrasonography in the upper extremity. Muscle Nerve 2013;47:864-71.

8. Yang SN, Yoon JS, Kim SJ, Kang HJ, Kim SH. Movement of the ulnar nerve at the elbow: a sonographic study. J Ultrasound Med 2013;32:1747-52.

9. Duncan I, Sullivan P, Lomas F. Sonography in the diagnosis of carpal tunnel syndrome. AJR Am J Roentgenol 1999;173:681-4.

10. Suh DH, Kim DH, Park JW, Park BK. Sonographic and electrophysiologic findings in patients with meralgia paresthetica. Clin Neurophysiol 2013;124:1460-4.

11. Zhu J, Zhao Y, Liu F, Huang Y, Shao J, Hu B. Ultrasound of the lateral femoral cutaneous nerve in asymptomatic adults. BMC Musculoskelet Disord 2012;13:227.

12. Damarey B, Demondion X, Boutry N, Kim HJ, Wavreille G, Cotten A. Sonographic assessment of the lateral femoral cutaneous nerve. J Clin Ultrasound 2009;37:89-95.

13. Boon AJ, Bailey PW, Smith J, Sorenson EJ, Harper CM, Hurdle MF. Utility of ultrasound-guided surface electrode placement in lateral femoral cutaneous nerve conduction studies. Muscle Nerve 2011;44:525-30.

14. el-Tantawi GA. Reliability of sensory nerve-conduction and somatosensory evoked potentials for diagnosis of meralgia paraesthetica. Clin Neurophysiol 2009; 120:1346-51. 